\title{
UTILIZAÇÃO DE GEOPROCESSAMENTO NA AGRICULTURA DE PRECISÃO
}

\author{
USE OF GEOPROCESSING IN PRECISION AGRICULTURE
}

\author{
Fernando Lucas Reghini - fernando.reghini@gmail.com \\ Fábio Alexandre Cavichioli - fabio.cavichioli@fatectq.edu.br \\ Faculdade de Tecnologia de Taquaritinga (Fatec) - Taquaritinga - São Paulo - Brasil
}

DOI: 10.31510/infa.v17i1.750

\section{RESUMO}

O aumento da competitividade tem transformado a agricultura, por muito tempo os produtores lidaram com o manejo de culturas sem armazenar as informações em bancos de dados, foi através do surgimento de novas tecnologias, como o geoprocessamento, que isso mudou, tornando possível a realização de comparações precisas com dados anteriores e com o histórico da área analisada. A agricultura de precisão é um conjunto de ferramentas e tecnologias que possibilita ao produtor conhecer toda a área para cultivo auxiliando no aumento do rendimento da produção. Esse modelo de agricultura tem auxiliado os profissionais da área, por meio de dados específicos, histórico de produtividade, aplicação e quantificação, fertilizantes utilizados, corretivos, com base na variabilidade espacial dos nutrientes no solo, auxiliando na tomada de decisões. O geoprocessamento é um conjunto de tecnologias destinadas a coletar e tratar dados espaciais com um objetivo específico. Este artigo tem como objetivo analisar a eficiência da utilização do geoprocessamento na agricultura de precisão.

Palavras-chave: Agricultura 4.0. Geotecnologia. Programas computacionais.

\begin{abstract}
Increased competitiveness has transformed agriculture, for a long time, producers dealt with crop management without storing information in databases, it was through the emergence of new technologies, such as geoprocessing, that this changed, making it possible to carry out accurate comparisons with previous data and with the history the analyzed area. Precision agriculture is a set of tools and technologies that allows the producer to get to know the entire area for cultivation, helping to increase production yield. This agricultural model has helped professionals in the area, through specific data, historical productivity, application and quantification, fertilizers used, corrective, based on the spatial variability of nutrients in the soil, helping in decision making. Geoprocessing is a set of technologies designed to collect and treat spatial data for a specific purpose. This article aims to analyze the efficiency of the use of geoprocessing in precision agriculture.
\end{abstract}

Keywords: Agriculture 4.0. Geotechnology. Computer Programs. 


\section{INTRODUÇÃO}

Nas últimas décadas ocorreram diversas transformações tecnológicas, fazendo com que as organizações alterassem seu ambiente em todos os níveis possíveis, resultando em uma reestruturação dos sistemas de gestão adotados, junto à análise da necessidade de implementação de tecnologias processuais (AUGUSTO et al, 2012).

Durante muito tempo a agricultura tradicional lidou com o manejo de culturas de modo desgastante, foi apenas a partir da utilização de novas tecnologias, como os dados georreferenciados, junto ao armazenamento de informações em bancos de dados que se tornou possível realizar comparações precisas com dados anteriores e com o histórico da área analisada.

A agricultura de precisão é um conjunto de ferramentas e tecnologias que possibilita ao produtor conhecer toda a área para cultivo de maneira mais completa e que pode ajudar a aumentar o rendimento da produção, nela o global é subdividido em pequenas frações homogêneas para que se alcance o máximo de rendimento de acordo com as potencialidades do solo e com o mínimo de poluição e degradação, sendo necessário o acompanhamento e gerenciamento de um volume muito grande de informações que variam no espaço e no tempo (ROCHA E LAMPARELLI, 1998) citado por ORLANDO et al. (1999).

A agricultura de precisão auxilia o agricultor e os profissionais da área a tomarem decisões, por meio de dados específicos, histórico de produtividade, aplicação e quantificação, fertilizantes utilizados, corretivos, com base na variabilidade espacial dos nutrientes no solo (MOLIN, 2003).

Há muito tempo agricultores têm buscado métodos inovadores para aperfeiçoar a sua propriedade, como a maximização da produtividade da cultura, o melhoramento genético, tecnologias aplicadas em correção e adubação do solo, variando a taxa de insumo de acordo com as características dos solos e do desempenho da cultura (COELHO, 2002).

Pode-se definir geoprocessamento como um conjunto de tecnologias destinadas a coletar e tratar dados espaciais com um objetivo específico. As atividades que envolvem o geoprocessamento são executadas pelos Sistemas de Informações Geográficas (SIG), que têm o objetivo de processar informações espaciais, sendo capazes de criar abstrações digitais do real, manejar e armazenar eficientemente dados, identificando o melhor relacionamento entre 


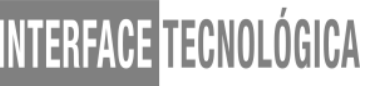

as variáveis espaciais e possibilitando a criação de relatórios e mapas para a compreensão desses relacionamentos.

É possível utilizar as ferramentas aplicadas na agricultura de precisão formando um conjunto de informações ou de modo individualizado, onde o agricultor pode adaptar as ferramentas e as tecnologias, adequando-as ao seu interesse e realidade financeira (MAPA, 2013).

O SIG organiza as informações por camadas temáticas e armazena de modo independente cada uma delas, permitindo um trabalho mais eficiente e o relacionamento de informações entre as diferentes camadas existentes (KORTE, 2001).

Dessa forma, o presente artigo tem como objetivo analisar a eficiência da utilização do geoprocessamento na agricultura de precisão, verificando as possibilidades de aplicação dessa ferramenta neste setor.

\section{FUNDAMENTAÇÃO TEÓRICA}

\subsection{Agricultura de Precisão}

$\mathrm{Na}$ atualidade, vários setores têm vivenciado transformações tecnológicas. Quando se fala em agricultura, planejar de modo adequado uma propriedade rural é uma tarefa indispensável para os produtores que desejam fazer o uso dessas terras. Oliveira (2003) entende que as organizações precisam repensar seus produtos e processos de produção, por meio de análises de satisfação dos clientes, de modo mais econômico ou pleno, devido ao aparecimento constante de novas tecnologias.

Para o Brasil, a agricultura é um fator de grande relevância na geração de riquezas, tendo se firmado como âncora do processo de estabilização dos preços e como fonte para obtenção de divisas (via exportações). Isso gerou alguns problemas como o aumento forçado das escalas de produção, a elevação dos custos acima das receitas, a redução dos preços recebidos, a compressão da renda agrícola, a concentração dos agentes compradores da produção agropecuária e a queda da renda da população consumidora (NUNES, 2007).

Sendo assim, desde o começo da década de 90 a agricultura brasileira vem sendo transformada, exemplos disso são as exportações de produtos agrícolas como estímulo ao aumento da produção, a concentração do controle do setor em mãos de grandes empresas 


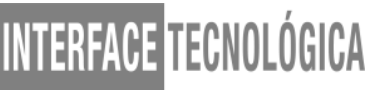

nacionais e transnacionais, a agricultura familiar e a competitividade das agroindústrias, a competitividade da agricultura familiar, a reforma agrária e, por fim, a produção de biocombustíveis. De acordo com Moreira e Rodrigues (2002) a inovação tecnológica é o principal fator do aumento da produtividade, além de ser importante recurso para elevar a capacidade de atuação das empresas na competição global e auxílio na conquista de novos mercados e consumidores.

Segundo Balastreire (1998), a agricultura de precisão pode ser definida como um conjunto de técnicas que permitem o gerenciamento localizado das culturas. A partir desse entendimento, é possível compreender a agricultura de precisão não apenas como uma prática cultural, mas como um modelo de gestão, englobando o uso de tecnologias para o manejo adequado das variações espaciais e fatores que afetam a produtividade (MANTOVANI, 2000).

A Agricultura de Precisão busca detectar, monitorar e manejar a variabilidade espacial e temporal dos sistemas de produção agropecuários visando otimizá-los. Apesar disso, os setores do agronegócio brasileiro vêm adotando a agricultura de precisão em um ritmo abaixo do previsto inicialmente (BERNARDI, 2011).

Esse sistema envolve conceitos de utilização de dados a respeito da variabilidade de propriedades locais e climáticas de determinada área, buscando aumentar a produtividade, otimizar o uso dos recursos e reduzir os impactos da agricultura ao meio ambiente. Os processos e as especificidades do solo que determinam o desempenho e a produção das culturas, e os impactos da agricultura ao meio ambiente, variam no espaço e no tempo, sendo assim, o conhecimento da variabilidade espacial e temporal dos fatores de produção da cultura é o passo inicial para a adoção, com êxito, do sistema de agricultura de precisão (RUNGE e HONS, 1999).

Coleta-se as informações da superfície terrestre por meio de um sensor remoto, com as radiações eletromagnéticas incidentes, e utilizando-se um software específico, é possível desenvolver cartas temáticas para um aproveitamento agrícola eficiente, podendo também monitorar a distância, já que as máquinas agrícolas também são influenciadas por mecanismos de precisão como o Global Positioning System (GPS) ou sistema de posicionamento global, assim, aproveitando melhor a colheita, o espaço, o tempo e economia gerada.

Apesar das variadas vantagens, a agricultura de precisão tem encontrado algumas dificuldades para sua implementação. De acordo com a EMBRAPA (2011) dentre essas 
dificuldades se encontram o alto custo dos equipamentos e a complexidade dos softwares, fazendo com que muitos produtores terceirizem para empresas privadas o levantamento das informações georreferenciadas. Já os prestadores de serviço em agricultura de precisão, apesar da expansão deste mercado, enfrentam dificuldade no acesso ao crédito para aquisição dos maquinários e dos equipamentos, limitando o seu crescimento e restringindo a tecnologia.

No entanto, Nunes (2012), entende que as principais dificuldades da implementação de práticas envolvendo a agricultura de precisão, relacionam-se a interpretação de um grande volume de dados, ao alto custo dos equipamentos, à adaptação das tecnologias às diferentes regiões existentes e a popularização das técnicas envolvidas no processo. Apesar da agricultura de precisão depender de um alto investimento inicial, seu custo-benefício é favorável para os produtores rurais, tendo diversas vantagens para quem busca melhor produtividade, diminuição de custos e maior competitividade com seus concorrentes.

\subsection{Geotecnologias}

De acordo com a EMBRAPA (2014), geotecnologias são conjuntos de técnicas e métodos científicos utilizados para a análise, exploração, estudo e conservação dos recursos naturais, considerando diferentes escalas e a informação espacial, sendo também utilizadas no estudo da paisagem e variáveis ambientais, na análise e auxílio na prevenção de desastres naturais, além de gerenciar e de monitorar a atividade humana. Esse conjunto de técnicas é formado por hardware (satélites, câmeras, GPS, computadores) e software capaz de armazenar e manipular as informações.

As geotecnologias abrangem tecnologias de processamento e armazenamento de dados geoespaciais por meio dos Sistemas de Informações Geográficas (SIG), Sistema de Navegação Global por Satélite (GNSS), e Sistemas de Processamento de Imagens (SPI). O nome mais correto para o Global Positioning System (GPS) é Navigation System for Timing and Ranging (NAVSTAR), esse sistema é composto por três segmentos: espacial (satélites), controle (rede de estações terrestres de monitoramento) e por fim, usuários.

Sensoriamento Remoto é a aquisição de informações a respeito de um objeto mediante análise de dados levantados por sensores remotos. Esses dispositivos conseguem coletar energia proveniente do objeto, convertê-la em sinal passível de ser registrado e apresentá-lo em forma adequada à extração de informações sobre o referido objeto. O Sistema de 


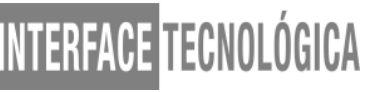

Informação Geográfica (SIG) é um tipo de geotecnologia que une hardware e software capazes de armazenar, analisar e processar dados georreferenciados. A análise das informações contidas auxilia na elaboração de mapas, gráficos, tabelas e relatórios que representam, digitalmente, a realidade (EMBRAPA, 2014).

Segundo Alonço et al. (2005), Veículo Aéreo Não Tripulado (VANT) são aeronaves pilotadas remotamente, que possuam algum tipo de aplicação científica, militar, dentre outras. São conhecidos popularmente como drones, sendo capazes de mapear áreas específicas e obter imagens de áreas de interesse com maior rapidez e custo reduzido em relação a outras tecnologias.

\subsection{Geoprocessamento}

O desenvolvimento tecnológico vem alcançando uma alta valorização, com a ciência buscando de modo geral satisfazer as demandas sociais. Logo, o meio tecnológico, em especial as tecnologias de informação, podem considerar-se atualmente como base para grande parte das atividades humanas. (BUZAI, 2004).

Fazem parte da geotecnologia o Processamento Digital de Imagens (PDI), a Geoestatística e os SIG. Tal conjunto de ferramentas e instrumentos delimitados por Silva (2003), reforça a ideia de que o entorno da geotecnologia é muito mais amplo do que se estabelece, não se limitando apenas a mapeamentos, localizações pontuais, dentre outros, e sim a um conjunto de fatores que levam a resultados almejados por diferentes profissionais.

Rodrigues (1993) define o geoprocessamento como o conjunto de tecnologias de coleta, tratamento, manipulação e apresentação de informações espaciais voltado para um objetivo específico. Este conjunto tem como ferramenta principal o Geographical Information System GIS, também conhecido como Sistema de Informações Geográficas (SIG).

É possível afirmar que conforme a tecnologia SIG tornou-se mais acessível, o planejamento evoluiu. O SIG permite uma avaliação mais ágil e objetiva, possibilitando o cruzamento de diferentes planos de informações para geração de mapas valiosos na avaliação de terras (FILHO et al., 2016).

O geoprocessamento associado às técnicas atuais de mapeamento, dentre elas a topografia convencional, utilização de fotografias aéreas, imagens de satélite, Sistema de Posicionamento Global por Satélite (GPS), imagens de vídeo, bem como outras formas de 
aquisição de dados, torna possível a aquisição de mapas temáticos e a quantificação de áreas, como por exemplo: áreas de agricultura, pastagem, campo nativo, reflorestamentos e florestamentos, florestas nativas (consideradas de preservação permanente), fruticultura, afloramentos rochosos, banhados, áreas sujeitas a alagamento, açudes, barragens, áreas erodidas ou em processos, comprimento de estradas e cercas, áreas degradadas, bem como outras formas de utilização (MOTTA; WATZLAWICK, 2000).

Rosa (2005), distingue SIG e geoprocessamento, afirmando que o geoprocessamento é um conceito mais abrangente e representa qualquer tipo de processamento de dados georreferenciados, enquando um SIG ou GIS processa dados gráficos e não gráficos (alfanuméricos) com ênfase em análises espaciais e modelagens de superfícies.

De acordo com Moreira, as ferramentas que são utilizadas no geoprocessamento constituem um conjunto denominado de Sistema de Informação Geográfica (SIG), o qual permite a interação do usuário com os dados obtidos.

Motta e Watzlawick (2000) afirmam que para fazer uso do geoprocessamento de maneira adequada é preciso possuir certo conhecimento dos recursos naturais como o solo, clima, vegetação, recursos hídricos, e da geografia da região como o relevo e a declividade. Também se faz necessário conhecer as características socioeconômicas para que se tenha um embasamento para identificação e utilização sustentada, ou desenvolvimento de determinada atividade apropriada, apontando a área que deva ser trabalhada ou preservada. Tendo em vista todo este volume de dados, é preciso que o produtor adote técnicas não convencionais para manipulação, planejamento e simulação dos dados, bem como para os levantamentos. Dentro deste contexto que o geoprocessamento e as técnicas de sensoriamento remoto demonstram grande potencial de aplicação.

O agricultor poderá relacionar a seu mapa um banco de dados, implantado em função da atividade desenvolvida na propriedade, visando o aumento da produtividade sem expandir a degradação ambiental. O geoprocessamento aumenta a eficiência na utilização dos insumos e a lucratividade da cultura, auxiliando o produtor na tomada de decisões, diminuindo o impacto ambiental da atividade agrícola, realizando o controle e acompanhamento no manejo de pastagens e solos, reflorestamentos e o monitoramento de áreas irrigadas, pragas e doenças. (MOTTA; WATZLAWICK, 2000). 


\section{PROCEDIMENTOS METODOLÓGICOS}

Foi realizada uma pesquisa bibliográfica por meio de artigos científicos, livros e pesquisas acadêmicas, com o intuito de analisar a utilização do geoprocessamento na agricultura de precisão.

\section{RESULTADOS E DISCUSSÃO}

O uso do geoprocessamento e a agricultura de precisão estão extremamente relacionados, produzindo diversas vantagens, dentre elas: a diminuição dos impactos ambientais da atividade agrícola, uma vez que a área explorada será menor, ocorrendo um manejo mais adequado e conservação do solo, aumento da eficiência entre utilização de insumos e lucratividade da cultura, melhor acompanhamento do produtor na tomada de decisões, monitoramento e controle de pragas e doenças em culturas. Dessa forma, a implantação de um sistema de geoprocessamento é essencial para se obter um planejamento adequado. Segundo Filho et al. (2016), o planejamento do uso das terras necessita da avaliação do seu potencial considerando as condições locais.

Uma das maiores dificuldades em relação ao geoprocessamento acaba sendo o alto custo dos softwares disponíveis, apesar de existirem alguns de domínio público. Outra dificuldade é a questão da qualificação dos profissionais que utilizarão essas tecnologias, sendo muitas vezes necessário a realização de treinamentos e o domínio de outros idiomas.

Castanho (2003) afirma que softwares de planejamento vêm se tornando essenciais para a realização da exploração econômica racional, tendo em vista que o aproveitamento econômico tem sido um fator indispensável para o desenvolvimento local, regional, nacional ou internacional.

As ferramentas do geoprocessamento são relevantes instrumentos usados para o levantamento de dados do espaço geográfico, propiciando um entendimento, mapeamento, interpretação, análise, correlações e planejamentos de áreas, dessa forma, é necessário compreender os conceitos do geoprocessamento junto a suas aplicações, buscando sua utilização pelos profissionais interessados (CASTANHO, 2006). 
De acordo com Machado et al. (2017) o grande número de dados georreferenciados, quando levantados em boa qualidade, permite que os SIG façam a espacialização destas informações, gerando produtos que subsidiam o planejamento e a tomada de decisão.

\section{CONSIDERAÇÕES FINAIS}

A atualidade tem sido marcada por uma grande competitividade entre as organizações, com a tecnologia sendo utilizada como vantagem competitiva para as empresas agrícolas. A agricultura de precisão tem desempenhado um papel de grande relevância para aumentar a competitividade, promovendo maior produtividade e qualidade, junto à redução do desperdício e da degradação ambiental.

O geoprocessamento, unido a agricultura de precisão, apresenta-se como uma relevante ferramenta de coleta, tratamento e análise de dados específicos, auxiliando o produtor de diversas formas, como por exemplo, no aumento da eficiência, produtividade, prevenção de pragas e doenças, dentre outros.

Por meio deste estudo, buscou-se levantar os benefícios da utilização do geoprocessamento na agricultura de precisão e de como essa ferramenta auxilia os produtores rurais. Foi possível constatar que com a utilização do geoprocessamento o produtor pode aumentar a produtividade e economizar nos custos da produção, mas vale ressaltar que apesar de estar se popularizando, muitos produtores encontram dificuldades em implementar tais ferramentas, devido ao alto custo de investimento, que envolve aquisição de equipamentos, softwares e treinamentos.

Apesar disso, o custo-benefício do geoprocessamento é grande, exigindo inicialmente certo investimento do produtor, mas produzindo em longo prazo diversas vantagens e benefícios nos âmbitos econômico e ambiental.

\section{REFERÊNCIAS}

ALONÇO, A. dos S. et al. Desenvolvimento de um veículo aéreo não tripulado (VANT) para utilização em atividades inerentes à agricultura de precisão. In: Congresso Brasileiro de Engenharia Agrícola, 35., 2005, Canoas. Anais... Jaboticabal: Associação Brasileira de Engenharia Agrícola, 2005. 
AUGUSTO, C. A. et al. A influência da inovação tecnológica na competitividade e nas relações de trabalho em usinas de açúcar e álcool paranaenses. Organizações Rurais \& Agroindustriais, Lavras, v. 14, n. 1, p. 1-14, 2012.

BALASTREIRE, L. A. A experiência com pesquisas em Agricultura de Precisão na ESALQ-USP. In: Congresso e feira para usuários de geoprocessamento da América Latina, 4., 1998, Curitiba. Anais... Curitiba: Microservice, 1998.

BERNARDI, A. Agricultura de Precisão: Um novo olhar. São Paulo: Embrapa, 2011. 11 p.

BUZAI, G. D. Geografia Global. El paradigma geotecnológico y

elespaciointerdisciplinarioenlainterpretacióndel mundo delsiglo XXI. $1^{\text {a }}$ impresión. Lugar Editorial. Buenos Aires. 224 p. ISBN 950-892-063-7, 2004.

CASTANHO, R. B. O processo de modernização agrícola e a utilização de técnicas de geoprocessamento na diferenciação físico - natural e ocupação em dois distritos no município de Palmeira das Missões/RS. 100 f. (Dissertação de Mestrado) - Centro de Ciências Rurais da Universidade Federal de Santa Maria - RS. 2003.

CASTANHO, R. B. Uso do geoprocessamento no estudo da produção agropecuária da microrregião geográfica de Carazinho - RS, 2006.

COELHO, A. M. Agricultura de Precisão: Manejo da Variabilidade Espacial e Temporal dos Solos e das Culturas. In: Tópicos em Ciência do Solo, Viçosa: SBCS, 2002.

FILHO, G. B. R.; FILHO, J. C. A.; CARVALHO, R. M. C. M. O.; ARAÚJO, M. S. B., FRUTUOSO, M. N. M. A.; BRANDÃO, S. S. F., 2016. Potencial agroecológico do Município de Itacuruba, Pernambuco, Brasil. Revista Brasileira de Geografia Física 9, p. 172-184.

EMBRAPA, Empresa Brasileira de Pesquisa Agropecuária. Geotecnologias e

Geoinformação: O produtor pergunta, a Embrapa responde. Brasília, DF, 2014. Disponível em: < https://ainfo.cnptia.embrapa.br/digital/bitstream/item/107363/1/500PGeotecnologias-e-geoinformacao-ed01-2014.pdf $>$. Acesso em: 20/03/2020.

KORTE, G. B. The GIS Book, How to Implement, manage, and asses the value of geographic information systems. Santa Fe: OnWorld Press, 2001.

MACHADO, T. C. E., Campos, M. C. C., Paganini, C. H. P., Maurício, J. C., Soares, M. D. R. Avaliação do uso e ocupação das áreas de preservação permanente nos anos de 2008 e 2013 na zona urbana de Humaitá, Amazonas. Revista Universidade Vale do Rio Verde 15, p. 744-750, 2017.

MANTOVANI, E.C.; QUEIROZ, D.M.; DIAS, G.P. Máquinas e operações utilizadas na agricultura de precisão. In: SILVA, F. M. da.(Coord.). Mecanização e agricultura de precisão. Poços de Caldas : UFLA/SBEA, 2000. p. 109-157. 
MAPA - Ministério da Agricultura, Pecuária e Abastecimento Secretaria de Desenvolvimento Agropecuário e Cooperativismo. Agricultura de precisão. Brasília: Brasil. Ministério da Agricultura, Pecuária e Abastecimento. 2013.

MOLIN, J.P. Agricultura de Precisão: situação atual e perspectivas. Piracicaba, p. 89-98, 2003.

MOREIRA, C. E.; RODRIGUES F. M. M. A Indústria e a Questão Tecnológica. Ministério da Ciência e Tecnologia, Finep, CNI , Brasília - DF, 2002.

MOREIRA, M. A. Fundamentos do sensoriamento remoto e metodologias de aplicações. São Jose dos Campos: INPE; 2001.

MOTTA, José LuisGaffrée; WATZLAWICK, Luciano Farinha .A importância do Geoprocessamento no Planejamento Rural. MundoGEO, Paraná. 2000. Disponível em: $<$ https://mundogeo.com/2000/02/02/a-importancia-do-geoprocessamento-no-planejamentorural>. Acesso em: 04 mar. 2020.

NUNES, José Luís da Silva. A Agricultura de Precisão como ferramenta para o produtor rural. Disponível em: $<$ https://www.agrolink.com.br/georreferenciamento/artigo/aagricultura-de-precisao-como-ferramenta-para-o-produtor-rural_82627.html >. Acesso em: 04 mar. 2020.

NUNES, S. O desenvolvimento da agricultura brasileira e mundial e a idéia de Desenvolvimento Rural.DESER, São Paulo, v. 1, n. 157, março. 2007. Disponível em: < http://www.deser.org.br/documentos/boletim_completo/Boletim157.pdf>. Acesso em: 04 mar. 2020.

OLIVEIRA, C. A. de. Inovação da tecnologia, do produto e do processo.Belo Horizonte: DesenvolvimentoGerencial, 2003.

ORLANDO, M. C.; CHENDRASEKHAR, A.; BUNDZ, S.; BURT, E. T.; MOORMAN, D. W.; TIMBERLAKE, G. A. The effect of peritoneal contamination on Wound Strength of small bowel and colonic anastomoses.AmSurg, Atlanta, v. 65, n. 7, p. 673-676, jul. 1999.

RODRIGUES, Marcos. Anais da quarta conferência latino-americana sobre sistemas de informação geográfica/segundo simpósio brasileiro de geoprocessamento. São Paulo: Epusp, 1993.

ROSA, R. Geotecnologias na Geografia aplicada. Revista do Departamento de Geografia, São Paulo, v. 16, p. 81-90, 2005.

RUNGE, E.C.A.; HONS, F.M. Precision agriculture- development of a hierarchy of variables influencing crop yields. In: international conference on precision agriculture, 4. , 1998. Proceedings... Minnesota: ASA-CSSA- SSSA, p. 143-158, 1999. 